\title{
Ageusia e Anosmia na Vigência de Tratamento com Leflunomida para Artrite Reumatóide (AR)
}

Aos Editores: O tratamento da artrite reumatóide (AR) tem como objetivo o alívio das queixas, a melhora da função e o ganho de qualidade de vida do paciente. A leflunomida, inibidor da síntese de pirimidina e da proliferação de linfócitos T CD $4^{(1)}$, tem sido uma opção para atingir tais objetivos. Entretanto, recentemente, vivenciamos o caso de uma paciente com AR tratada com leflunomida, que apresentou alteração na sensibilidade gustativa e olfatória, efeitos colaterais ainda não descritos na literatura. Assim, L.S.V.M., 62 anos, branca, feminina, do lar, portadora de AR há sete anos, conforme critérios do American College of Rheumatology (ACR), iniciou tratamento com leflunomida no terceiro ano da sua enfermidade nas doses usuais, totalizando 40 meses de uso. Ao iniciar a ingestão da mesma, a paciente apresentava poliartrite, acometendo punhos, joelhos e tornozelos, sem manifestações extra-articulares, fator reumatóide $=233(<40)$ e classe funcional II, segundo critérios do $\mathrm{ACR}^{(2)}$. Esta opção terapêutica, associada à baixa dose de corticóide oral - deflazacorte equivalente a $4 \mathrm{mg}$ de prednisona/dia - fez regredir a doença para classe funcional I em 60 dias e normalização de parâmetros laboratoriais. Após o quinto mês de tratamento, houve relato de alteração gustativa e olfatória, caracterizando ageusia e anosmia. A paciente relatava incapacidade de distinguir o sabor dos alimentos e de identificar cheiros e perfumes. Apesar do aparecimento desses efeitos colaterais, optamos por manter a medicação, justificada pelos bons resultados clínicos obtidos. $\mathrm{O}$ uso da mesma foi interrompido pela paciente quando estava com 40 meses de tratamento, pois sentia-se bem. Após três meses sem o medicamento, houve recuperação total da sensibilidade gustativa e olfatória. Em contrapartida, neste período, retornaram as queixas

\section{REFERÊNCIAS}

1. MacDonald J, Zhong T, Lazarescu A, Gan BS, Harth M. Vasculitis associated with the use of leflunomide. J Rheumatol 31: 2076-2078, 2004.

2. Hochberg MC, Chang RW, Dwosh I, Lindsey S, Pincus T, Wolfe F. The American College of Rheumatology 1991 Revised Criteria for the Classification of Global Functional Status in Rheumatoid Arthritis. Arthritis Rheum 35: 498-502, 1992. inflamatórias articulares e a VHS (velocidade de hemossedimentação) aumentou para $41 \mathrm{~mm}$.

A leflunomida apresenta efetividade terapêutica e segurança semelhantes às da sulfassalazina e do metotrexato ${ }^{(3)}$. Os efeitos colaterais mais comumente vistos em pacientes em uso da leflunomida são diarréia, elevação de enzimas hepáticas, alopecia, rash e elevação da pressão arterial, efeitos estes não apresentados pela paciente ${ }^{(3,4,5)}$. Diversas drogas têm sido associadas a alterações gustativas e olfatórias ${ }^{(6)}$. O presente relato é o primeiro caso destas alterações em uma paciente fazendo uso de leflunomida, conforme a pesquisa feita em livros de referência em reumatologia, no PubMed e Medline, entre 1993-2005. Nenhum dos efeitos colaterais descritos na literatura, referentes ao uso da leflunomida, ocorreram com esta paciente. Houve total recuperação da sensibilidade gustativa e olfatória, o que significou ganho de qualidade de vida à paciente, que optou por não fazer mais uso da leflunomida, embora tenham retornado as queixas articulares.

Apesar do relato deste caso, os autores não hesitam em considerar a leflunomida uma opção terapêutica para a AR, considerando que este efeito colateral não foi grave, ainda que tenha afetado órgãos de sentido.

Após a suspensão da leflunomida, substituiu-se o fármaco previamente em uso por outra DMARD.

Marcelo Campos Appel da Silva Vicente Sperb Antonello Acadêmicos de Medicina da Universidade Luterana do Brasil (ULBRA), Canoas-Porto Alegre, RS;

Fernando Appel da Silva Médico Reumatologista do Complexo Hospitalar Santa Casa de Misericórdia de Porto Alegre, RS.

3. Osiri M, Shea B, Robinson V, et al. Leflunomide for the Treatment of Rheumatoid Artritis: A Systematic Review and Metaanalysis. J Rheumatol $30: 1182-1190,2003$

4. Kremer JM, Cannon GW. Benefits / risk of leflunomide in rheumatoid arthritis. Clin Exp Rheumatol 22: S95-100, 2004

5. O'Dell JR. Therapeutic Strategies for Rheumatoid Arthritis. New England Journal of Medicine 17, 350 : 2591-2602, 2004.

6. Mann NM, Lafreniere D. Anatomy and etiology of taste and smell disorders. Up to Date, 1999 Nov. 